\title{
T-20 and T-1249 HIV fusion inhibitors' structure and conformation in solution: a molecular dynamics study ${ }^{\ddagger}$
}

\author{
ANTÓNIO M. T. MARTINS DO CANTO, ${ }^{a, b}$ A. J. PALACE CARVALHO, ,a,b J. P. PRATES RAMALHO ${ }^{a, b}$ and LUÍS \\ M. S. LOURA ${ }^{\mathrm{b}, \mathrm{c} *}$ \\ a Departamento de Química, Universidade de Évora, Portugal \\ b Centro de Química de Évora, Portugal \\ c Faculdade de Farmácia, Universidade de Coimbra, Portugal
}

Received 27 June 2007; Revised 3 October 2007; Accepted 22 October 2007

\begin{abstract}
Fusion of the HIV envelope with the target cell membrane is a critical step of the HIV entry into the target cell. Several peptides based on the C-region of HIV gp41 have been used in clinical trials as possible HIV fusion inhibitors. Among these are T-1249 and T-20 (also known as enfurvitide). Despite recent works, a detailed molecular picture of the inhibitory mechanism of these molecules is still lacking. These peptides are usually depicted as $\alpha$-helices by analogy with the structure of the sequence of the gp41 protein with which they are homologous. However, structures like these would be highly unstable in solution and thus would not explain, by themselves, the ability that the two fusion inhibitors have to become solvated by water and also interact effectively with cell membranes. To this effect, extensive molecular dynamics simulations were carried out to investigate the structure and conformational behavior of T-1249 and T-20 in water, as well as shorter homologous peptides CTP and 3f5, which show no inhibitory action. We found that the studied inhibitors have no stable structure in solution in the time scale studied. Additionally, the solvent accessible area varies significantly during the simulation. Our findings suggest that these peptides may assume not only one, but several possible sets of structures in solution, some of which more adequate to interact with the solvent, whereas others might be better suited to interact with cell membranes. Interestingly, and in accordance with published experimental studies, we verified that T-1249 displays considerably larger $\alpha$-helical structure than T-20. Taking into account a recent study with design peptides with increased helicity, it is possible that this feature may be related to the increased inhibiting efficiency of T-1249 relative to that of T-20. Copyright @ 2007 European Peptide Society and John Wiley \& Sons, Ltd.
\end{abstract}

Keywords: AIDS; HIV fusion inhibitor; enfurvitide; T-20; T-1249; molecular dynamics; molecular simulations

\section{INTRODUCTION}

Membrane fusion between enveloped viruses and host cell membranes is an obligatory process of viral infection that is mediated by viral glycoproteins [1-3]. The initial steps of HIV-1 infection of a target cell (binding of the virus to the target cell and fusion of the membranes of both) are mediated by the viral envelope glycoprotein complex formed by the transmembrane protein gp4l (responsible for membrane fusion), and the surface protein gp120 (responsible for host recognition), bound to the external domain of gp41 [4]. Entry of the HIV into a target cell is performed in three steps: (i) binding of the viral gp120 to the target cell surface protein CD4 (a protein present in helper T-cells and involved in the immunologic response), a critical step for the attachment of the virus particle to the target cell [5]; (ii) this process changes the conformation of gp120 enabling this protein to bind to yet another receptor on the immune system cell's

\footnotetext{
*Correspondence to: Luís M. S. Loura, Faculdade de Farmácia, Universidade de Coimbra, Rua do Norte, 3000-295 Coimbra, Portugal; e-mail: lloura@ff.uc.pt

$\$$ This article is part of the Special Issue of the Journal of Peptide Science entitled "2nd workshop on biophysics of membrane-active peptides".
}

surface, typically CCR5 [6] or CXCR4 [7]; and (iii) gp41mediated membrane fusion [4]. The previous processes, mediated by gp120, result in a conformational change in gp41, which exposes a fusion peptide and allows it to insert into the membrane of the target cell, hence leading to the fusion of the two membranes and the entry of the viral components into the target cell $[4,8]$. Several peptides based on the C-region of the HIV gp41 have been used in clinical trials as possible HIV fusion inhibitors [reviewed in [9]]. Among these are T-1249 [10] and T-20, also known as enfurvitide [11-13] (Figure 1).

$\mathrm{T}-20$ is a synthetic 36 -amino-acid peptide whose sequence is homologous to the $C$-terminal of HR2 (Heptad Repeat 2) of gp41 [11]. This first generation peptide is currently one of the more advanced clinical drugs for inhibiting HIV-1 entry and has received fast Food and Drugs Administration (FDA) approval [14,15]. Despite T-20's effectiveness it has, already, encountered some resistant strains of HIV $[12,15]$.

T-1249 is a synthetic 39-amino-acid peptide composed of sequences derived from HIV-1, HIV-2, and Simian immunodeficiency virus (SIV) [10]. This fusion inhibitor is in phase II clinical trials, and both phase I and II clinical trials have shown that it is a stronger inhibitor of HIV entry than T-20 and it retains function against T-20 resistant strains [10,12,13]. 


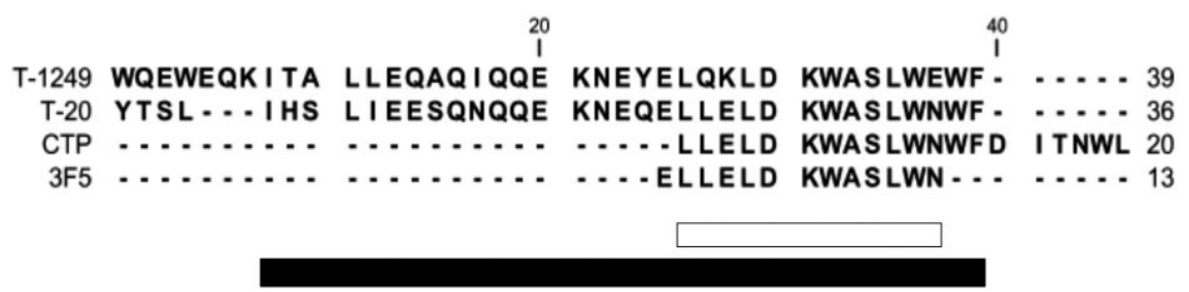

Figure 1 Sequence comparison of the four peptides under study. Homology between all the peptides (black bar) and between T-20 and T-1249 (white bar) is highlighted.

Despite recent works, a detailed molecular picture of the inhibitory mechanism promoted by these molecules is still lacking. Working models for their mechanisms of action, based mainly on fluorescence spectroscopy data, were presented in [16] (T-20) and [17] (T-1249). Both schemes rely on the experimentally verified efficient partition of the two peptides to zwitterionic bilayers, and differences between them arise from the possibility of T-1249 (but not T-20) adsorbing to cholesterol-rich membranes, which might be the main cause of its improved efficiency (see Ref. 17 for a detailed discussion).

T-20 and T-1249 are usually depicted, at least partly, as $\alpha$-helices by analogy with the structure of the sequences with which they are homologous. However, this representation is most probably not accurate for an aqueous environment. The presence of a large number of hydrophobic amino-acid residues in the sequence of both peptides (Figure 1) would cause them, in solution, to fold in order to form more globular structures that would improve their solubility. This fact has been observed for both T-20 and T- 1249 by CD spectroscopy [17]. These peptides also have the ability to interact with/partition to cell membranes and their mode of action depends partly on that property. However, both fusion inhibitors in this study are endovenous drugs and, as such, knowledge of their structure and behavior in solution would help our understanding of their mode of action.

In this work, we study the structure and behavior of these two peptides in solution using MD simulations in the $100 \mathrm{~ns}$ time scale. This was done also with the aim of determining models of the two fusion inhibitors that could be used to study their interaction with model membranes. Two smaller model peptides, $3 f 5$ and CTP (Figure 1), homologous with both fusion inhibitors in study, were also investigated with the same approach. The use of smaller peptides allows for longer simulated time ranges and hence for the study of longer time scale properties. In this way, $3 \mathrm{f5}$ and CTP were studied to determine whether the studied 100-ns time range was sufficient or if there was the need to extend the study to longer time ranges.

\section{METHODS}

Initial models of the four peptides ( $\alpha$-helices) were built with the Arguslab 4.01 package [18] and solvated in cubic simulation boxes with SPC water [19], with dimensions and water content proportional to the size of the peptide in question (the distance between each molecule and the box walls is $0.5 \mathrm{~nm}$ ): $3 \mathrm{f5}$ was simulated in a box of $3.45 \times 3.45 \times 3.45 \mathrm{~nm}^{3}$ with 1278 water molecules, CTP in a box of $4.54 \times 4.54 \times 4.54 \mathrm{~nm}^{3}$ with 2964 water molecules, T-20 in a box of $7.19 \times 7.19 \times 7.19 \mathrm{~nm}^{3}$ with 12160 water molecules, and T-1249 in a box of $7.41 \times 7.41 \times 7.41 \mathrm{~nm}^{3}$ with 13330 water molecules. The Gromacs 3.3.1 simulation package was used to perform all the MD simulations [20,21]. The force field used to describe the peptide interactions was the Gromos96 43al force field $[22,23]$. Prior to the MD simulation, the four systems underwent a steepest-descent energy minimization of the structure, followed by a small MD run with restraints in the peptide atoms positions to properly allow the solvent molecules to adjust/relax around the peptide. Restraints were subsequently removed, and extensive (100 ns) molecular dynamics simulations were then performed under constant number of particles, pressure ( 1 bar), and temperature ( $300 \mathrm{~K})$, and using periodic boundary conditions. Pressure and temperature control was carried out using the weakcoupling Berendsen schemes [24], with coupling times of 1.0 and $0.1 \mathrm{ps}$, respectively. Isotropic pressure coupling was used. All bonds were constrained to their equilibrium values, using the SETTLE algorithm [25] for water and the LINCS algorithm [26] for all other bonds. This approach allowed the use of a time-step of $2 \mathrm{fs}$. Cutoffs of $1 \mathrm{~nm}$ were used in the treatment of electrostatic interactions. Although this is not the most accurate treatment for this type of long range interactions, it allowed the study of longer time scales in a more reasonable computer simulation time. The monitored parameters include the root mean square deviation (RMSD) relative to the initial conformation, radius of gyration (RG), secondary structure (SS), and solvent accessible surface (SAS). RMSD and RG were calculated using Gromacs 3.3.1 analysis packages [20, 21], whereas SS and SAS were computed using the DSSP program [27].

\section{RESULTS AND DISCUSSION}

In the time scale studied, all the peptides evolve roughly in a similar fashion from the $\alpha$-helix initial model to a more or less globular flexible structure. This evolution occurs in two stages. In the first stage ( $t<25 \mathrm{~ns}$ ), the peptides rapidly change in conformation. This is clearly 
shown in the time dependence of the RMSDs which have a larger variation in this time frame (Figure 2), thus suggesting this rapid structural change. The RGs, as a measure of the size of the peptide, also have a substantial decrease in this first stage (Figure 3), thus reflecting a change from an $\alpha$-helix to a more globular structure. All peptides evolve away from the initial models into globular structures (see Figure 4 for typical snapshots of these) that are more capable of protecting the more hydrophobic residues from the solvent, therefore stabilizing the structure in solution. Comparing T-20 with T-1249, it can be seen from Figure 4 that the changes in secondary structure away from the $\alpha$-helical initial model are especially rapid in the former. This is a first indication of the experimentally verified [17] increased helicity of T-1249 relative to $\mathrm{T}-20$ (but see also below).

This first stage of the folding of these peptides is followed by a second stage $(t>\sim 25)$ of smoother evolution in which they adopt a number of different globular flexible structures that interchange between one another (see snapshots and SS evolution in Figure 4). This is also apparent in the time variations of the RMSD (Figure 2), RG (Figure 3), and SAS (Figures 5 and 6) for all peptides, with the exception of CTP. For the latter molecule, these properties suffer larger variations in the first $25 \mathrm{~ns}$ and afterwards fluctuate around an average value. In comparison with the other peptides, the RMSDs plot of CTP shows smaller fluctuations (Figure 2), and it also shows a considerable helical content. This different behavior may be related to the additional six residues located in its $C$-terminal (Figure 1) that may be helping the its structure to stabilize and form the $\alpha$-helix. By comparison, 3f5, which does not possess these residues, behaves in much the same way as the other peptides, with the exception of the T-1249's $N$-terminus behavior that also shows moderate helical content. This behavior seems to be important, since it was observed in a recent report that helix-stabilized HIV-1 fusion inhibitor peptides

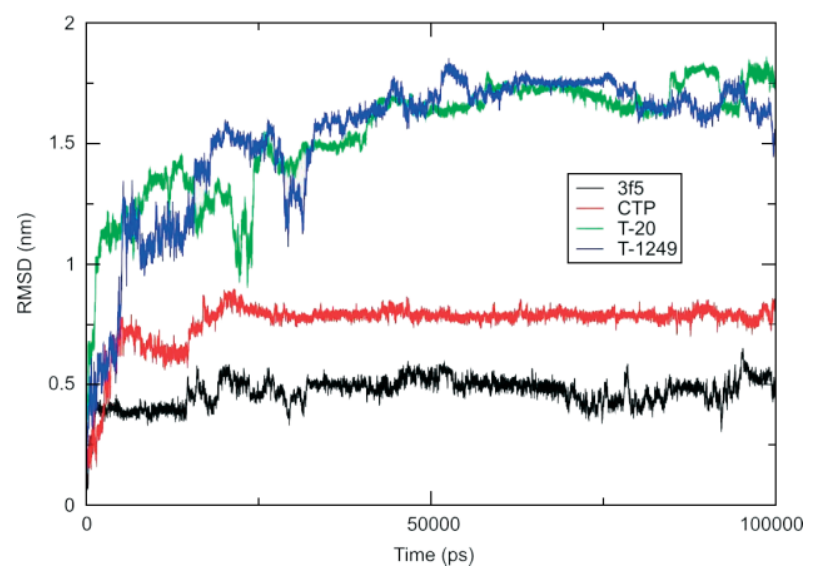

Figure 2 Root mean square deviation relative to each model's initial conformation ( $\alpha$-helix structures in all cases).

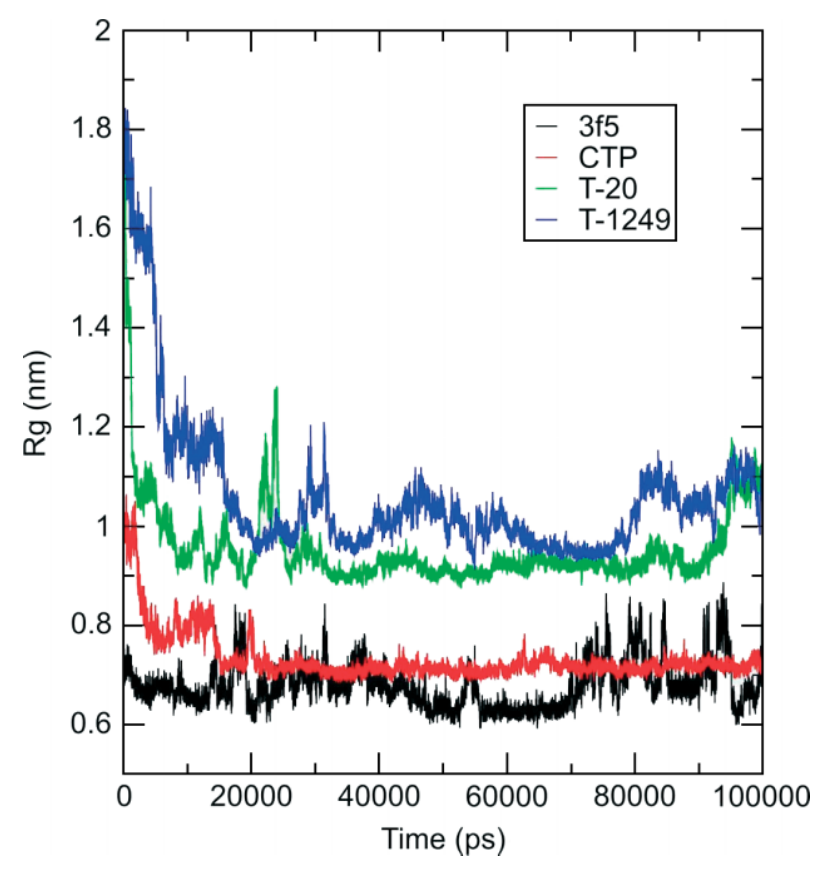

Figure 3 Radius of gyration as a function of time for each peptide under study.

were more active than T-20 - as much as 3600-fold [28]. However, further studies with a wider variety of peptides would be required to better understand these results. In particular, the absence of inhibitory activity in CTP in spite of the stability of its helical structure (and 14-amino-acid homology with T-20), suggests that this is not the single factor influencing the peptide's inhibitory activity.

To investigate if the lack of a stable structure for the fusion inhibitors and $3 f 5$ in our simulations was due to a limitation in the time scale simulated, we carried out longer simulations of $3 \mathrm{f5}$ (500 ns). No change in the described structural pattern was apparent, ruling out time scale problems in this matter.

Both the SAS of each residue (Figure 5) and the SS of T-20 and T-1249 (Figure 4) vary significantly during the simulation time. The average content of $\alpha$-helix for the last $75 \mathrm{~ns}$ of each simulation of these peptides was calculated and was found to be negligible for T-20 and $16.4 \%$ for $\mathrm{T}-1249$ in qualitative accordance with experimental data obtained by Veiga and coworkers by CD spectroscopy measurements [17]. These authors measured 8 and $30 \%$ of $\alpha$-helical content in buffer, for T-20 and T-1249, respectively. The higher results obtained in their study may be related to the intrinsic differences between the recovery of secondary structure from CD spectra (only three types of structures are recognized [29]) and the SS analysis of our atomistic MD simulations (which differentiates 8 types of secondary structures [27]). An alternative explanation is that the studied time scale is insufficient for secondary structure analysis, and that structural changes may occur in longer time scales. 

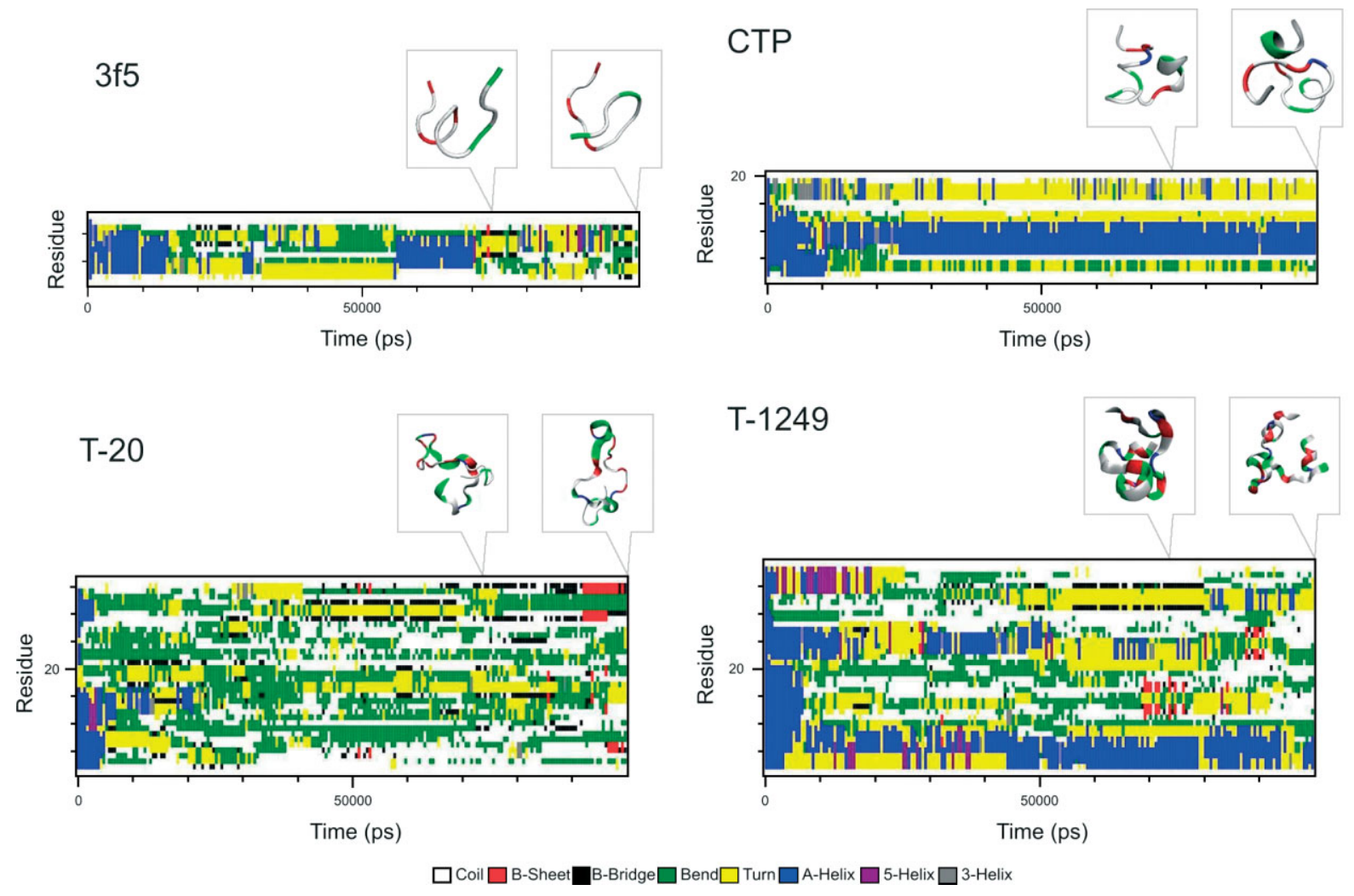

Figure 4 Secondary structure as a function of time of the four peptides under study. Insets: snapshots of each peptide's model structure taken at 75 and $100 \mathrm{~ns}$.
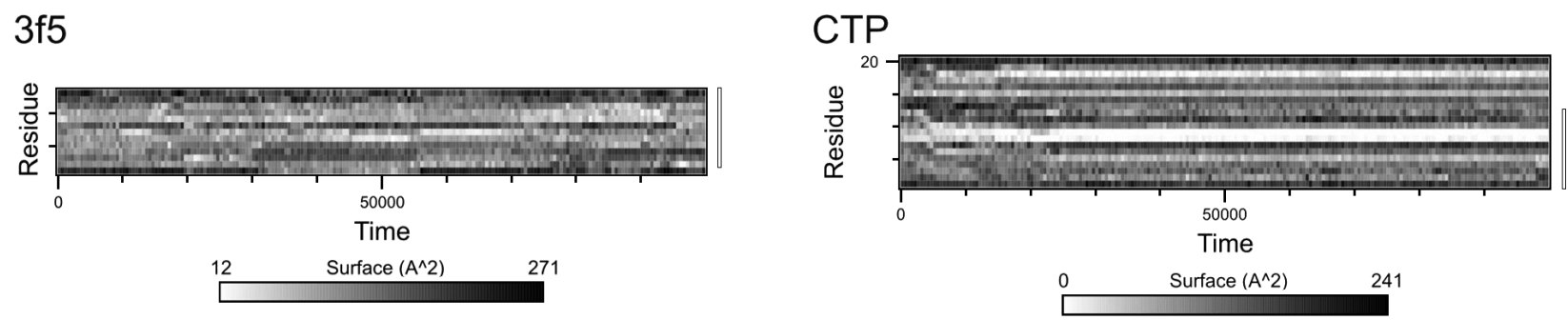

\section{$\mathrm{T}-20$}
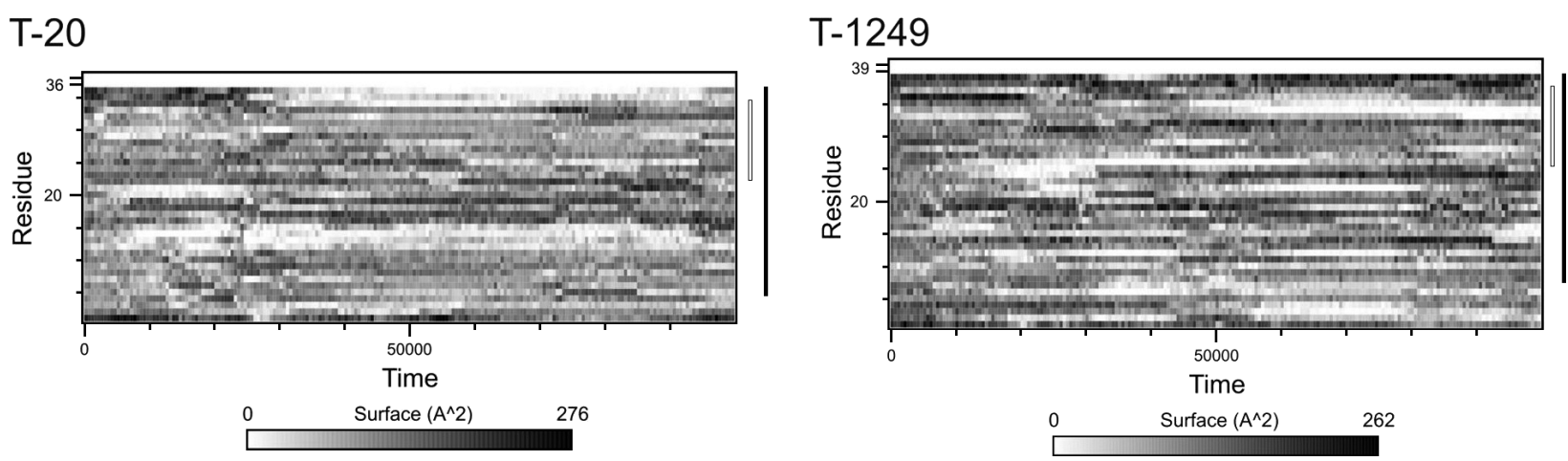

Figure 5 Solvent accessible surface as a function of time for each residue. Homology between peptides is highlighted as in Figure 1.

\section{CONCLUSIONS}

Our results show that the studied peptides have no stable structure in solution in the studied time scale, and also that these peptides, especially the fusion inhibitors, may assume not only one, but several possible sets of structures in solution, some of which are probably extensively solvated, whereas 


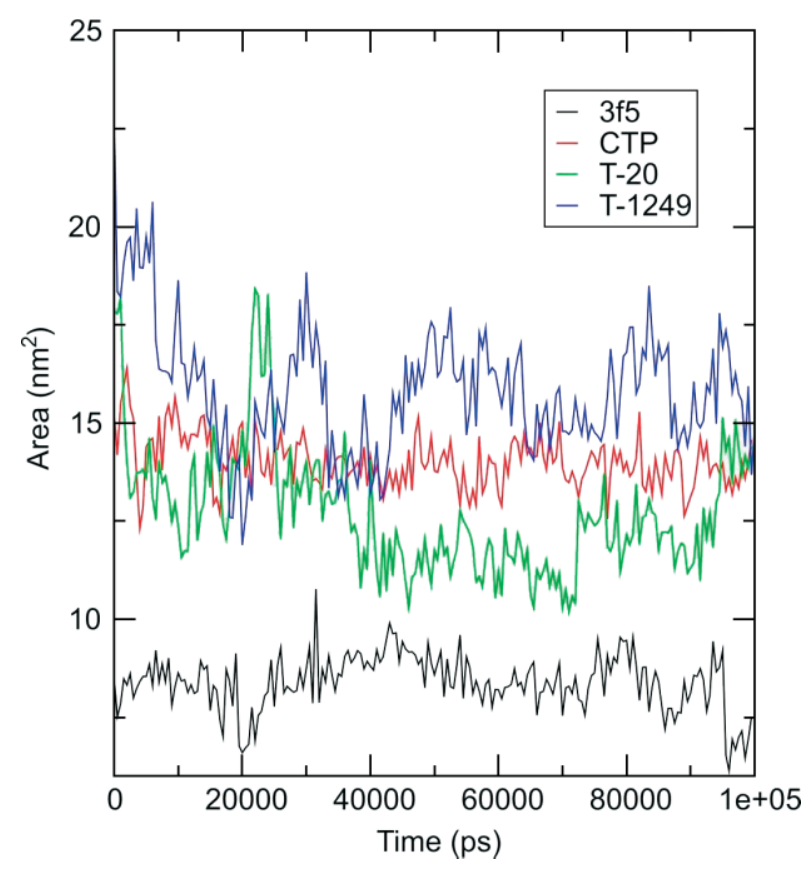

Figure 6 Total solvent accessible surface as a function of time, for each peptide under study.

others might be better suited to interact with cell or model membranes. These properties suit the inhibitor peptides to the function they were designed for, as they should be sufficiently soluble, but still capable of interacting, adsorbing and/or incorporating into lipid membranes. Molecular models of the interactions of both T-20 and T-1249 with model membranes were proposed by Veiga and coworkers $[16,17]$. Their results show that both T-20 and T-1249 are able to partition to the host membrane. Translocation should be impossible because of charge effects. Furthermore, T-1249 should be able to interact more strongly with cholesterol-rich surfaces, such as lipid rafts, where HIV-1 receptor CD4 and coreceptors CXCR4/CCR5 are considered to be located [30] (this may account for its higher efficiency in inhibiting the fusion of the virus, as suggested in [17]). To evaluate these hypotheses, simulations of the interactions of the fusion inhibitor peptides with model membranes of varying composition are underway. One of the main goals of the present study was to obtain realistic models of the inhibitory peptides in aqueous solution that could be used as starting points for simulations of the peptides in presence of model membranes. In this way, $\mathrm{T}-1249$ is currently being simulated in the presence of a 1-palmitoyl-2-oleoyl-sn-glycero-3-phosphocholine bilayer. Using the final structure of the peptide in this study as starting point, located in water $\sim 2 \mathrm{~nm}$ above the lipid/water interface, a preliminary observation of the trajectory under calculation points to a rapid adsorption of the peptide to the surface of the model membrane ( $t<4-5 \mathrm{~ns}$; data not shown). The possibility of effective peptide incorporation for longer times is being investigated.

\section{Acknowledgments}

Funding from POCI and PPCDT projects, FCT, Portugal, is acknowledged.

\section{REFERENCES}

1. Weissenhorn W, Dessen A, Calder LJ, Harrison SC, Skehel JJ, Wiley DC. Structural basis for membrane fusion by enveloped viruses. Mol Membr Biol. 1999; 16: 3-9.

2. Fujii G, Horvath S, Woodward S, Eiserling F, Eisenberg D. A molecular model for membrane fusion based on solution studies of an amphiphilic peptide from HIV gp41. Protein Sci. 1992; 1: 1454-1464.

3. Lawless MK, Barney S, Guthrie KI, Bucy TB, Petteway SR Jr, Merutka G. HIV-1 membrane fusion mechanism: structural studies of the interactions between biologically-active peptides from gp41. Biochemistry 1996; 35: 13697-13708.

4. Colman PM, Lawrence MC. The structural biology of type I viral membrane fusion. Nat. Rev. Mol. Cell Biol. 2003; 4: 309-319.

5. Lasky LA, Nakamura G, Smith DH, Fennie C, Shimasaki C, Patzer E, Berman P, Gregory T, Capon DJ. Delineation of a region of the human immunodeficiency virus type 1 gp120 glycoprotein critical for interaction with the CD4 receptor. Cell 1987; 50: 975-985.

6. Dragic T, Litwin V, Allaway GP, Martin SR, Huang Y, Nagashima KA, Cayanan C, Maddon PJ, Koup RA, Moore JP, Paxton WA. HIV-1 entry into CD4+ cells is mediated by the chemokine receptor CC-CKR-5. Nature 1996; 381: 667-673.

7. Feng Y, Broder CC, Kennedy PE, Berger EA. HIV-1 entry cofactor: functional cDNA cloning of a seven-transmembrane, G proteincoupled receptor. Science 1996; 272: 872-877.

8. Kliger Y, Aharoni A, Rapaport D, Jones P, Blumenthal R, Shai Y. Fusion peptides derived from the HIV type 1 glycoprotein 41 associate within phospholipid membranes and inhibit cell-cell Fusion. Structure-function study. J. Biol. Chem. 1997; 272: 13496-13505.

9. Jiang S, Zhao Q, Debnath AK. Peptide and non-peptide HIV fusion inhibitors. Curr. Pharm. Des. 2002; 8: 563-580.

10. Eron JJ, Gulick RM, Bartlett JA, Merigan T, Arduino R, Kilby JM, Yangco B, Diers A, Drobnes C, DeMasi R, Greenberg M, Melby T, Raskino C, Rusnak P, Zhang Y, Spence R, Miralles GD. Short-term safety and antiretroviral activity of T-1249, a second-generation fusion inhibitor of HIV. J. Infect. Dis. 2004; 189: 1075-1083.

11. Kilby JM, Hopkins S, Venetta TM, DiMassimo B, Cloud GA, Lee JY, Alldredge L, Hunter E, Lambert D, Bolognesi D, Matthews T, Johnson MR, Nowak MA, Shaw GM, Saag MS. Potent suppression of HIV-1 replication in humans by T-20, a peptide inhibitor of gp41-mediated virus entry. Nat. Med. 1998; 4: 1302-1307.

12. Baldwin CE, Sanders RW, Berkhout B. Inhibiting HIV-1 entry with fusion inhibitors. Curr. Med. Chem. 2003; 10: 1633-1642.

13. Cooley LA, Lewin SR. HIV-1 cell entry and advances in viral entry inhibitor therapy. J. Clin. Virol. 2003; 26: 121-132.

14. Kilby JM, Lalezari JP, Eron JJ, Carlson M, Cohen C, Arduino RC, Goodgame JC, Gallant JE, Volberding P, Murphy RL, Valentine F, Saag MS, Nelson EL, Sista PR, Dusek A. The safety, plasma pharmacokinetics, and antiviral activity of subcutaneous enfuvirtide (T-20), a peptide inhibitor of gp41-mediated virus fusion, in HIV-infected adults. AIDS Res. Hum. Retroviruses 2002; 18: $685-693$.

15. Kilby JM, Eron JJ. Novel therapies based on mechanisms of HIV-1 cell entry. N. Engl. J. Med. 2003; 348: 2228-2238.

16. Veiga S, Henriques S, Santos NC, Castanho M. Putative role of membranes in the HIV fusion inhibitor enfuvirtide mode of action at the molecular level. Biochem. J. 2004; 377: 107-110.

17. Veiga AS, Santos NC, Loura LMS, Fedorov A, Castanho MA. HIV fusion inhibitor peptide T-1249 is able to insert or adsorb to lipidic bilayers. Putative correlation with improved efficiency. J. Am. Chem. Soc. 2004 ; 126: 14758-14763. 
18. Thompson MA. ArgusLab 4.0.1. Planaria Software LLC: Seattle, WA, 2004.

19. Berendsen HJC, Postma JPM, van Gunsteren WF, Hermans J. Interaction models for water in relation to protein hydration. In Intermolecular Forces, Pullman B (ed.). D. Reidel Publishing Company: Dordrecht, 1981; 331-342.

20. Bekker H, Berendsen HJC, Dijkstra EJ, Achterop S, van Drunen R, van der Spoel D, Sijbers A, Keegstra H, Reitsma B, Renardus MKR. GROMACS: A parallel computer for molecular dynamics simulations. In Physics Computing 92, de Groot RA, Nadrchal J (eds). World Scientific: Singapore, 1993.

21. van der Spoel D, Lindahl E, Hess B, Groenhof G, Mark AE, Berendsen HJC. GROMACS: fast, flexible and free. J. Comp. Chem. 2005; 26: 1701-1718.

22. van Gunsteren WF, Daura X, Mark AE. The GROMOS force field. In Encyclopaedia of Computational Chemistry, Schleyer PvR, Allinger L, Schaefer F (eds), John Wiley and Sons: New York, 1997.

23. Scott WRP, Hunenberger PH, Tironi IG, Mark AE, Billeter SR, Fennen J, Torda AE, Huber T, Kruger P, van Gunsteren WF. The GROMOS biomolecular simulation program package. J. Phys. Chem. A 1999; 103: 3596-3607.

24. Berendsen HJC, Postma JPM, van Gunsteren WF, DiNola A, Haak JR. Molecular dynamics with coupling to an external bath. $J$. Chem. Phys. 1984; 81: 3684-3690.
25. Miyamoto S, Kollman PA. SETTLE: an analytical version of the SHAKE and RATTLE algorithm for rigid water models. J. Comp. Chem. 1992; 13: 952-962.

26. Hess B, Bekker H, Berendsen HJC, Fraaije JGEM. LINCS: A linear constraint solver for molecular simulations. J. Comp. Chem. 1997; 18: $1463-1472$.

27. Kabsch W, Sander C. Dictionary of protein secondary structure: pattern recognition of hydrogen-bonded and geometrical features. Biopolymers 1983; 22: 2577-2637.

28. Dwyer JJ, Wilson KL, Davison DK, Freel SA, Seedorff JE, Wring SA, Tvermoes NA, Matthews TJ, Greenberg ML, Delmedico MK. Design of helical, oligomeric HIV-1 fusion inhibitor peptides with potent activity against enfuvirtide-resistant virus. Proc. Natl. Acad. Sci. U.S.A. 2007; 104: 12772-12777.

29. Andrade MA, Chacón P, Merelo JJ, Morán F. Evaluation of secondary structure of proteins from UV circular dichroism using an unsupervised learning neural network. Prot. Eng. 1993; 6: 383-390.

30. Campbell SM, Crowe SM, Mak J. Virion-associated cholesterol is critical for the maintenance of HIV-1 structure and infectivity. AIDS 2002; 16: 2253-2261. 